\title{
New Wavelet Neurovascular Bundle for Bedside Evaluation of Cerebral Autoregulation and Neurovascular Coupling in Newborns with Hypoxic-Ischemic Encephalopathy
}

\author{
Lina F. Chalak Rong Zhang \\ Department of Pediatrics and Internal Medicine, University of Texas Southwestern Medical Center, Dallas, TX, USA
}

\section{Keywords}

Neurovascular coupling · Hypothermia .

Near-infrared spectroscopy · Amplitude-integrated

electroencephalography

\begin{abstract}
Neonatal encephalopathy (NE) resulting from birth asphyxia constitutes a major global public health burden for millions of infants every year, and despite therapeutic hypothermia, half of these neonates have poor neurological outcomes. As new neuroprotective interventions are being studied in clinical trials, there is a critical need to establish physiological surrogate markers of therapeutic efficacy, to guide patient selection and/or to modify the therapeutic intervention. The challenge in the field of neonatal brain injury has been the difficulty of clinically discerning NE severity within the short therapeutic window after birth or of analyzing the dynamic aspects of the cerebral circulation in sick NE newborns. To address this roadblock, we have recently developed a new "wavelet neurovascular bundle" analytical system that can measure cerebral autoregulation (CA) and neurovascular coupling (NVC) at multiple time scales under dynamic, nonstationary clinical conditions. This wavelet analysis may allow noninvasive quantification
\end{abstract}

\section{KARGER}

(C) 2017 S. Karger AG, Basel

E-Mail karger@karger.com

www.karger.com/dne at the bedside of (1) CA (combining metrics of blood pressure and cerebral near-infrared spectroscopy, NIRS) and (2) NVC (combining metrics obtained from NIRS and EEG) in newborns with encephalopathy without mathematical assumptions of linear and stationary systems. In this concept paper, we present case examples of NE using the proposed physiological wavelet metrics of CA and NVC. The new approach, once validated in large NE studies, has the potential to optimize the selection of candidates for therapeutic decision-making, and the prediction of neurocognitive outcomes.

(c) 2017 S. Karger AG, Basel

\section{Introduction}

Neonatal encephalopathy (NE) resulting from birth asphyxia constitutes a major global public health burden for millions of infants every year, and despite therapeutic hypothermia, half of these neonates have poor neurological outcomes $[1,2]$. There is a pressing need to accurately identify $\mathrm{NE}$ in a timely fashion due to the limited therapeutic window of $6 \mathrm{~h}$ after birth for the initiation of neuroprotective interventions. The asphyxia insult impairs fetal cerebral blood flow (CBF) and is manifested postna-

Lina F. Chalak, MD, MSCS

University of Texas Southwestern Medical Center at Dallas Department of Pediatrics, Division of Neonatal-Perinatal Medicine 5323 Harry Hines Boulevard, Dallas, TX 75390-9063 (USA)

E-Mail lina.chalak@utsouthwestern.edu 
tally by a distinctive NE, which is usually classified after birth using the clinical modified Sarnat stages as mild, moderate and severe NE. Recent reports suggest new evidence of cognitive impairment in a subset of $30-50 \%$ of "mild NE" cases, who currently cannot be identified early on and therefore not offered therapy $[3,4]$.

The challenge in the field of neonatal brain injury has been the difficulty of clinically discerning NE severity within the short therapeutic window after birth, or alternatively of analyzing the dynamic, nonstationary aspects of the cerebral circulation in sick NE newborns. An improved NE stratification on the first day of life would facilitate individualized and effective neuroprotective therapies targeting those newborns at higher risk while sparing those at lower risk the adverse effects of therapies. In general, brain injury studies have focused mostly on serum and CSF biomarkers assessed at discrete time points. These biomarkers are limited by the lack of real-time analysis, independent validation in large cohorts, and, as such, have not translated into clinical practice in NE [58]. We therefore propose a new concept of a "wavelet neurovascular (NV) bundle" approach defined by a new wavelet analysis system to dynamically quantify cerebral autoregulation (CA) and NV coupling (NVC) in real time and discuss case examples of its application to improve the stratification of NE severity after birth.

\section{Why Do We Need to Measure CA and NVC in Real Time: The NV Unit Concept}

The NV unit consists of a comprehensive communication between neurons, glia, endothelial cells, pericytes, astrocytes, and basement membranes. These functions are critical in the delivery of oxygen/nutrients and isolating the brain from circulatory disturbances $[9,10]$. These critical functions are impaired by asphyxia and are difficult to clinically evaluate at the bedside [11]. The cerebral vasculature is exquisitely sensitive to perfusion pressure, and the brain requires a steady delivery of oxygen and glucose to maintain brain health and function. Thus, CA reflects the ability of the cerebral vasculature to maintain a relatively steady blood flow in the face of changes in perfusion pressure $[12,13]$. In addition, intrinsic neuronal oscillating activities have been described in the cortical neurons across mammalian species at multiple time scales [14]. These oscillations span from very low to ultrafast frequency bands and facilitate synaptic plasticity [15] and brain functional connectivity-revealing brain neuronal functionality $[14,16,17]$. Therefore intact dynamic CA and NVC functions, based on hemodynamic and neural activity oscillations, appear paramount to survival and normal functions.

\section{CA: Gaps in Knowledge regarding Available Methodology}

CA was initially viewed as a nonchanging phenomenon maintaining constant $\mathrm{CBF}$ over a range of perfusion pressures $[12,18]$. However, the recent development of CBF measures with high temporal resolution has allowed for rapid beat-to-beat measurements and revealed the dynamic characteristics of CA [19]. Animal studies further demonstrate the dynamic nature of CA and show a spectrum of autoregulatory impairment with hypoxia [20], hypercarbia [21], and acidosis by invasive positron emission tomography (PET) studies [22]. With these results, it became clear that CA should be measured as a continuous variable rather than a dichotomous yes/no description. The current traditional methodology briefly reviewed below includes using either a time domain or a frequency-based transfer function approach, each of which have certain limitations.

Transfer function analysis can assess the dynamic properties of CA intermittently, by measuring coherence (i.e., the correlation between changes in arterial pressure and $\mathrm{CBF}$ velocity), gain (i.e., the amplitude), and phase (i.e., the time relationship) [23-25]. These studies demonstrated that CA is most operational at very low frequencies $<0.07 \mathrm{~Hz}$ [23]. The analysis is however based on the assumption of a stationary time series and works only under such conditions [26]. These mathematical assumptions of nonchanging stationary variables are unlikely to be maintained in sick NE during the $72 \mathrm{~h}$ of hypothermia therapy [27].

Time domain analysis $[28,29]$ using a linear Pearson correlation is suited for sick newborns, as it assesses CA over long durations by using a predetermined window, but is limited in capturing the dynamic aspect as the size of window of correlation may influence CA estimates [30].

The challenges specific to NE are numerous. Although the approaches described above have each been utilized in hypoxic-ischemic encephalopathy (HIE) and correlated with outcomes [31,32], the trade-off of time versus frequency has been a limiting step. In a recent pilot study of 20 newborns with NE undergoing hypothermia, we have reported large spontaneous fluctuations in blood pressure (BP; range $30 \pm 4 \mathrm{~mm} \mathrm{Hg}$ ), associated with impaired cerebral hemodynamics [33]. Time domain cor-
90

Dev Neurosci 2017;39:89-96 DOI: $10.1159 / 000457833$
Chalak/Zhang 
relation analysis between changes in perfusion (i.e., arterial pressure and cerebral near-infrared spectroscopy, NIRS, as proxy of CBF) was performed. We tested multiple time scale moving correlations, because the moving time window could influence the scale of the changes in CA. We demonstrated in newborns with NE different patterns of impaired CA across different time scales which were associated with abnormal outcomes. In-phase or positive correlations were detected at short time scales of $10 \mathrm{~min}$. Separate antiphase or negative correlations were detected at longer time scales of $120 \mathrm{~min}$. Both of the patterns of positive and negative correlations were associated with Bayley scores $<85$ at 24 months (ROC curves: AUC 0.75, 95\% CI 0.4-0.95). Physiologically, the positive correlations indicate the pressure passive circulation, while the inverse correlation at the long time scale suggests a reperfusion phenomenon, which has been described before in translational piglet's asphyxia models in that tested range [34-36]. These newly described complex time scale variations specific to HIE represented a measurement challenge using the approaches mentioned above which are not adapted to (1) measure in real time such complex correlations, (2) identify select positive or negative correlations, or (3) simultaneously cover the full wide range of time frequencies in NE.

\section{NVC: Gaps in Knowledge regarding Available Methodology}

Roy and Sherrington [37] introduced the NVC concept over a century ago. CBF has subsequently been shown to vary in relation to the cerebral metabolic rate of both glucose and oxygen, and tight coupling of cerebral function, metabolism, and blood flow is well established and has been demonstrated in numerous physiological, biochemical, and clinical studies $[38,39]$. The hyperemic response to neuronal activation occurs within seconds, appears to be mediated by astrocytes and is highly localized [39]. NVC is currently measured by modern-day neuroimaging studies of brain function with PET and functional MRI in adults $[17,40,41]$. However, such techniques cannot be applied in a fragile newborn nor can they measure the multifrequency aspect of NVC. These techniques do not permit a real-time analysis of continuous dynamics, because of the susceptibility to movement artifact.

To date, coupling has only been described in healthy newborns within 1 frequency band using time correlations of EEG and NIRS oscillations [42]. However, it is the more complex slow rhythms which have not been mea-

Wavelet Neurovascular Bundle surable noninvasively in newborns that synchronize large spatial domains and affect connectivity and repair, and are likely to be impaired with NE [43].

\section{New Wavelet NV Bundle Method of Analysis}

The challenge of physiological measures of CA and NVC is the presence of dynamic and frequency-dependent variables, which impedes the utility of the traditional analysis methods. Conceptually, a method to allow monitoring the comprehensive functions of the NV unit via biomediators measured in real time could provide a paradigm shift to the field of neonatal neuroprotection. The wavelet mathematical analysis has been demonstrated to provide an optimal representation of signals in the time scale domain and has traditionally been more confined to the field of natural sciences rather than medicine [44]. Conceptually, it represents the ideal framework to study nonstationary signals in medicine, as it can quantify the coherence and phase between 2 time series as a function of both time and frequency scale. We have adapted this wavelet analysis to develop a new wavelet NV bundle to measure CA (by correlating BP and NIRS changes) and NVC (by correlating amplitude-integrated electroencephalography, aEEG, and NIRS changes). The analysis is not limited to a specific monitoring device but can be adapted to a multitude of noninvasive devices (such as continuous BP monitors, NIRS, and aEEG) that are all available at the bedside. While high NIRS cerebral saturation and suppressed aEEG background neuronal activity have been each separately linked to abnormal outcomes in HIE [45, 46], we have integrated aEEG-NIRS wavelet signals to quantify the degree of NVC to measure changes in brain vascular function and its relation to neuronal electrical activity. We first describe the methodology of the new wavelet NV bundle analysis and then provide illustrative case examples. Figure 1 is an example of the visual wavelet output, which, depending on the 2 variables selected, can allow noninvasive quantification at the bedside of (1) CA (the 2 selected variables are BP and cerebral NIRS), and (2) NVC (the 2 selected variables are NIRS and EEG). Our bioengineering team has developed a Matlab program to directly calculate the significant coherence over any study time period [47]. Mathematically, strength of the correlation is calculated as percentage of significant coherences using statistical simulations $(p<$ 0.05 ). The area under the curve can be determined for the significant coherences. Further, the maximum duration of impairment in CA/NV can be calculated [47].

Dev Neurosci 2017;39:89-96 DOI: $10.1159 / 000457833$ 
Fig. 1. Wavelet multiple time-scale visual representation. a Wavelet coherence characterizes cross-correlations between 2 selected physiological variables as a function of a wide range of both time and frequencies [44]. Time is represented on the $x$ axis, while the $y$ axis on the right depicts the frequencies in hertz, and on the left the corresponding scale in minutes. The $R^{2}$ coherence index is in red color and represents the significant correlations across the time-frequency axis that can be visually assessed or numerically translated into percent coherence. The color bar shows coherence from 0 to 1 , where 0 is the least coherence (blue) and 1 is the highest coherence (red), while the arrows within the red area indicate either the direction of the correlations or sign (negative: $\leftarrow$, positive: $\rightarrow$ ). All red area output per study duration can be quantified to percent coherence using MATLAB. b Cartoon depicting the proposed wavelet $\mathrm{NV}$ bundle in newborns with encephalopathy which can measure CA and NVC depending on the choice of the 2 variables. MAP, mean arterial pressure.

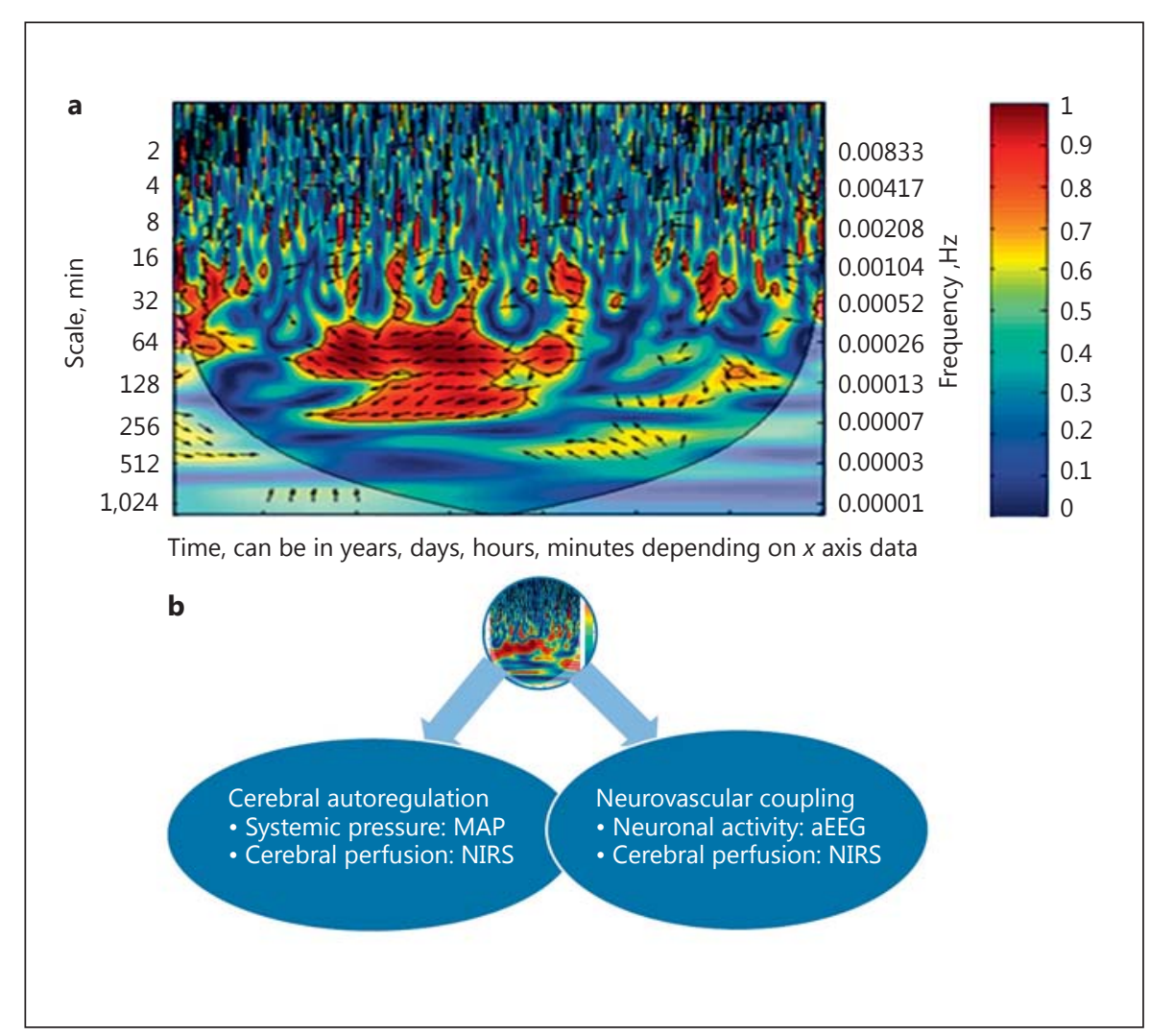

\section{Methodology Summary of CA Wavelet}

Intra-arterial BP is continuously measured from an indwelling umbilical arterial catheter. Regional cerebral tissue oxygen saturation $\left(\mathrm{SctO}_{2}\right)$ is measured on the frontoparietal side of the neonate's head using an INVOSTM 4100-5100 oximetry (Somanetics, Troy, MI, USA) neonatal sensor. Frontoparietal NIRS leads are usually selected because this area is representative of the global watershed region and is most affected by asphyxia. Both mean arterial pressure and $\mathrm{SctO}_{2}$ data are sampled at a rate of 2 data points/min and recorded synchronously with a Vital Sync ${ }^{\mathrm{TM}}$ system (Somanetics Corporation, Troy, MI, USA). The mean arterial pressure and $\mathrm{SctO}_{2}$ data are collected under stable conditions $\left(\mathrm{pCO}_{2} 40-50 \mathrm{~mm} \mathrm{Hg}\right.$ and hemoglobin $12-15 \mathrm{mg} / \mathrm{dL})$.

\section{Methodology Summary of NVC Wavelet}

aEEG leads are placed at the standard C3/P3-C4/P4 along with the overlapping frontoparietal NIRS leads to assess NVC, because this area is representative of the global watershed region and is most affected by asphyxia. The signals are amplified and bandpass-filtered $(1-50 \mathrm{~Hz})$ to minimize artifacts and electrical interferences and rec- tified as per aEEG manufacturing. The raw EEG filtered voltage is used to calculate bandwidth: the difference between upper and lower margins for the selected P3-P4 channel is quantitatively calculated using Brainz Analyze Research (v1.5) software (Natus Medical). Peak-to-peak bandwidth amplitudes are measured using a moving average every $0.5 \mathrm{~s}$. The wavelet is used to measure coherences between the simultaneously recorded EEG bandwidth and cerebral NIRS. Figures 2 and 3 represent case examples demonstrating the wavelet method to visualize and quantify the exact percentage of CA and NVC, respectively, as compared to the descriptive approach that is usually obtained from such devices.

\section{Is the Wavelet NV Bundle Methodology Ready for Prime Time Yet?}

The cases summarized using the new methodology illustrate a potentially important impact of the wavelet in improving the stratification of NE severity for selection of candidates for therapies, and providing early physiological markers of outcome predictions. 


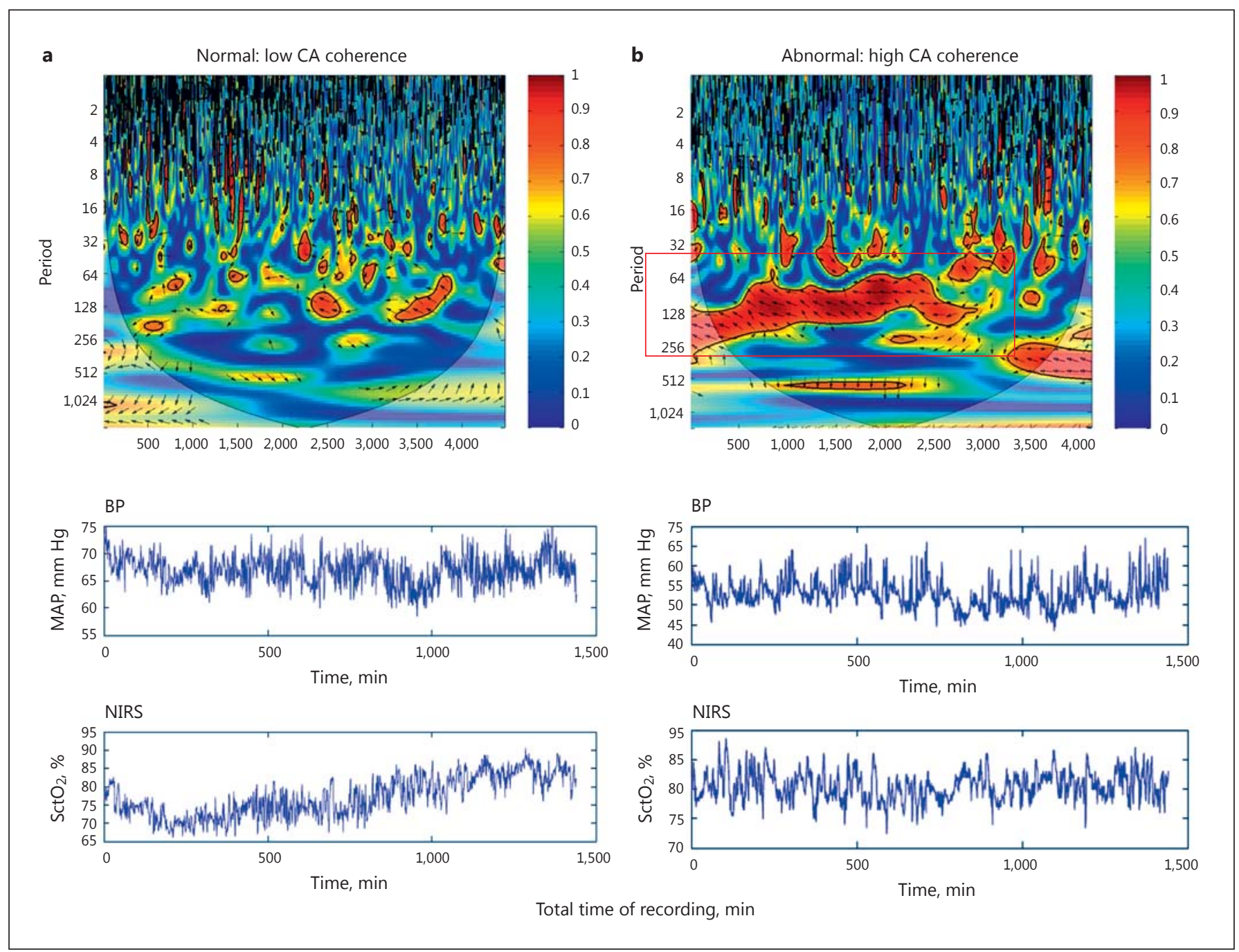

Fig. 2. Cases highlighting the analysis of CA during hypothermia therapy. Simultaneous raw data on continuous BP and regional cerebral $\mathrm{SctO}_{2}$ are plotted over time during hypothermia therapy with normal (a) and abnormal neurodevelopmental outcomes at 24 months (b). The selective output from each of the NIRS and BP is seen in the lower panel. The wide range of BP (50-70) and NIRS (70-85) for each of the selected variables during the $66 \mathrm{~h}$ of steady state provides limited information by itself. The wavelet allows the representation of CA changes over time as seen in red. Significant

The strengths of this methodology include (1) a precise and simultaneous quantification of CA and NVC, across a wide range of time and frequency scales, (2) a potential for real-time analysis once fully validated and developed, and (3) adaptability to a multitude of devices which can be synchronized in time. The methodology has limitations with respect to (1) the sampling frequencies of the selected instruments, (2) the overall time of

Wavelet Neurovascular Bundle in-phase coherence (highlighted in box) between $\mathrm{SctO}_{2}$ and aEEG is seen in a very low-frequency range of $0.00025-0.001 \mathrm{~Hz}$. Using the MATLAB program in the moderate NE with normal outcome (a), the CA coherence was calculated to be $27 \%$, whereas in the severe NE with abnormal outcomes (b), the CA coherence was calculated to be $52 \%$ of the total recording time, capturing both positive and negative coherences. As a reference, the coherence was $<10 \%$ during the same time period in the noncooled control (not visualized).

recording (a minimum of $4 \mathrm{~h}$ is needed), the length of which should be comparable across subjects compared, and (3) the need of a steady-state recording, in order to meet assumptions needed for providing proxies of $\mathrm{CBF}$ and metabolism. Among the available devices to monitor cerebral perfusion, NIRS cerebral oximetry is favored due to its noninvasiveness, instrument portability, and ease of use. However, a limitation of most NIRS is the 


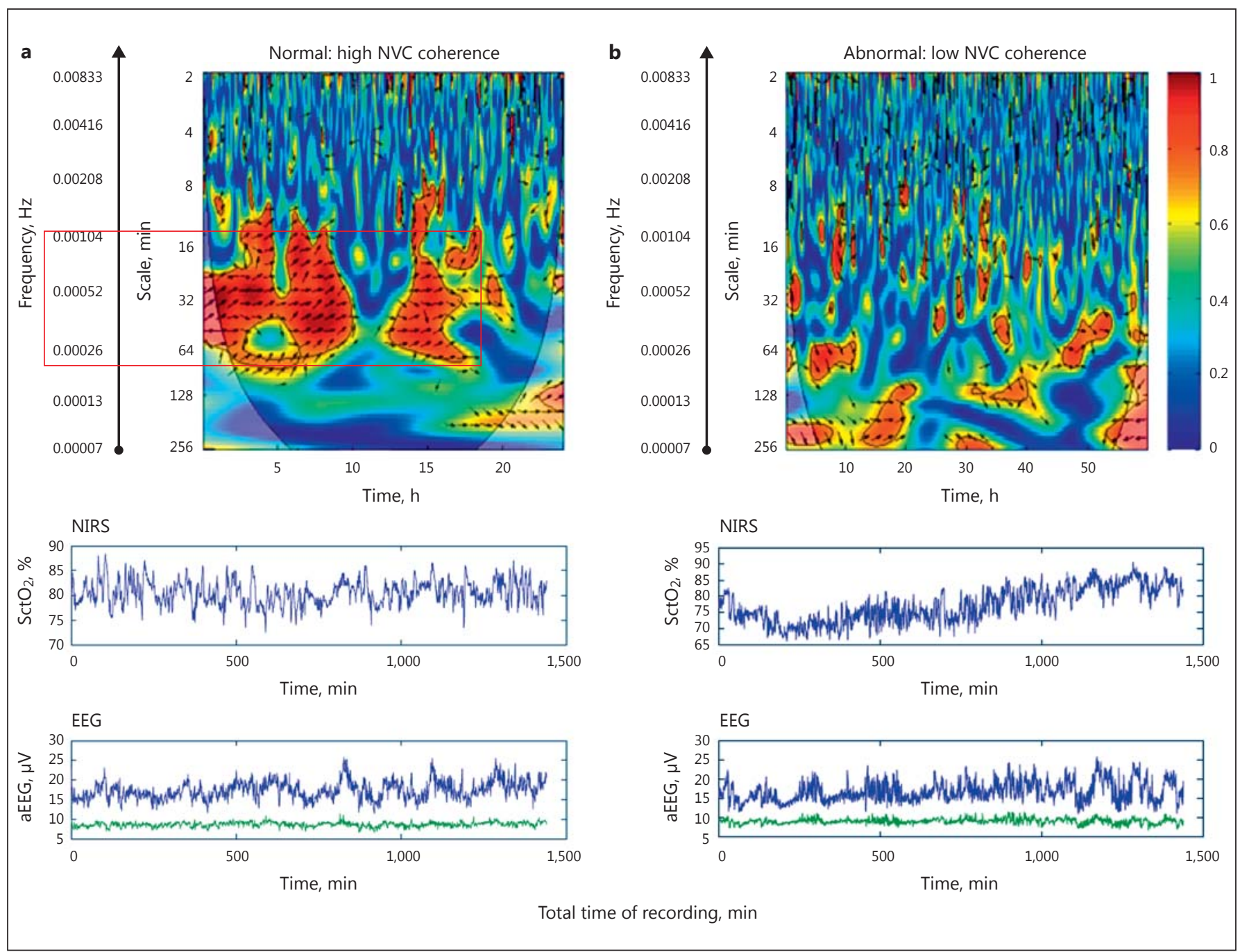

Fig. 3. Example of NVC in NE after birth. Simultaneous aEEG and regional cerebral $\mathrm{SctO}_{2}$ are plotted over time to indicate NVC with normal outcomes (a) and with abnormal neurodevelopmental outcomes at 24 months (b). A different NVC pattern is demonstrated by the wavelet output below. a This extract is an example of NVC output from a normal outcome in a neonate with no encephalopathy and a normal outcome (MRI showed no injury and all Bayley III domains were $\geq 85$ ). Significant coherence is shown in the red box at very low frequency $(0.00026-$

lack of detail in the signal output of which cerebral oxygenation is derived, thereby necessitating steady-state analysis with stable oxygenation and normative values of hemoglobin and $\mathrm{CO}_{2}$. We do not know either how the findings apply to the preterm infant as hemodynamic responses to neuronal activation appear to be incompletely developed in the neonatal and infant brain, compared with the adult brain [48].
$0.001 \mathrm{~Hz}$ ). MATLAB calculated the NVC\% as $45 \%$ during the recording. b This infant is an example of abnormal NVC, as the stage of NE worsened from mild to moderate around $24 \mathrm{~h}$. The NVC coherences calculated by MATLAB were less than $5 \%$, indicating impaired NVC during the first $48 \mathrm{~h}$ of life in an infant who subsequently developed an abnormal outcome. MRI showed evidence of white matter injury. The Bayley III scores at 18 months were abnormal: cognitive 71 , language 70 , and motor 85 .

In conclusion, the NV wavelet bundle has direct applicability as it captures the dynamic aspects of the magnitude and duration of impaired cerebral hemodynamics such as CA and NVC, in relation to outcomes in NE, and is currently being validated in large cohorts. The knowledge obtained is likely to impact the risk stratification strategies, therapeutic decision-making, and prediction of long-term neurocognitive outcomes in NE after birth.
94

Dev Neurosci 2017;39:89-96 DOI: $10.1159 / 000457833$
Chalak/Zhang 
An important remaining key gap in knowledge is whether maintaining the cerebral hemodynamic parameters within a "normal" range would translate into improved outcomes in HIE. This analytical approach using the wavelet NV bundle may provide a basis for a novel precision medicine approach, which has been lacking in the HIE neuroprotective trials to date.

\section{Acknowledgment}

Dr. Chalak is supported by NIH grant K23HD069521.

\section{References}

1 Bryce J, Boschi-Pinto C, Shibuya K, Black RE: WHO estimates of the causes of death in children. Lancet 2005;365:1147-1152.

2 Pryds O, Greisen G, Lou H, Friis-Hansen B: Vasoparalysis associated with brain damage in asphyxiated term infants. J Pediatr 1990; 117:119-125.

3 Dupont TL, Chalak LF, Morriss MC, Burchfield PJ, Christie L, Sanchez PJ: Short-term outcomes of newborns with perinatal acidemia who are not eligible for systemic hypothermia therapy. J Pediatr 2013;162:35-41.

4 Murray DM, O'Connor CM, Ryan CA, Korotchikova I, Boylan GB: Early EEG grade and outcome at 5 years after mild neonatal hypoxic ischemic encephalopathy. Pediatrics 2016; 138:e20160659.

5 Mir IN, Chalak LF: Serum biomarkers to evaluate the integrity of the neurovascular unit. Early Hum Dev 2014;90:707-711.

6 Chalak LF, Tarumi T, Zhang R: The "neurovascular unit approach" to evaluate mechanisms of dysfunctional autoregulation in asphyxiated newborns in the era of hypothermia therapy. Early Hum Dev 2014;90:687-694.

7 Chalak LF, Sanchez PJ, Adams-Huet B, Laptook AR, Heyne RJ, Rosenfeld CR: Biomarkers for severity of neonatal hypoxic-ischemic encephalopathy and outcomes in newborns receiving hypothermia therapy. J Pediatr 2014;164:468-474.e1.

8 Chalak LF: Inflammatory biomarkers of birth asphyxia. Clin Perinatol 2016;43:501-510.

9 Quillinan N, Herson PS, Traystman RJ: Neuropathophysiology of brain injury. Anesthesiol Clin 2016;34:453-464.

10 Kratzer I, Chip S, Vexler ZS: Barrier mechanisms in neonatal stroke. Front Neurosci 2014;8:359.

11 Moretti R, Pansiot J, Bettati D, Strazielle N, Ghersi-Egea JF, Damante G, Fleiss B, Titomanlio L, Gressens P: Blood-brain barrier dysfunction in disorders of the developing brain. Front Neurosci 2015;9:40.

12 Lassen NA: Cerebral blood flow and oxygen consumption in man. Physiol Rev 1959;39: 183-238.

13 Buckley EM, Parthasarathy AB, Grant PE, Yodh AG, Franceschini MA: Diffuse correlation spectroscopy for measurement of cerebral blood flow: future prospects. Neurophotonics 2014;1:011009.
14 Buzsaki G, Freeman W: Editorial overview: brain rhythms and dynamic coordination. Curr Opin Neurobiol 2015;31:v-ix.

15 Butefisch CM: Plasticity in the human cerebral cortex: lessons from the normal brain and from stroke. Neuroscientist 2004;10:163-173.

16 Owen JP, Li YO, Yang FG, Shetty C, Bukshpun P, Vora S, Wakahiro M, Hinkley LB, Nagarajan SS, Sherr EH, Mukherjee P: Resting-state networks and the functional connectome of the human brain in agenesis of the corpus callosum. Brain Connect 2013;3:547562.

17 Tarapore PE, Findlay AM, Lahue SC, Lee H, Honma SM, Mizuiri D, Luks TL, Manley GT, Nagarajan SS, Mukherjee P: Resting state magnetoencephalography functional connectivity in traumatic brain injury. J Neurosurg 2013;118:1306-1316.

18 Lou HC, Lassen NA, Friis-Hansen B: Impaired autoregulation of cerebral blood flow in the distressed newborn infant. J Pediatr 1979;94:118-121.

19 Tan CO, Taylor JA: Integrative physiological and computational approaches to understand autonomic control of cerebral autoregulation. Exp Physiol 2014;99:3-15.

20 Tweed A, Cote J, Lou H, Gregory G, Wade J: Impairment of cerebral blood flow autoregulation in the newborn lamb by hypoxia. Pediatr Res 1986;20:516-519.

21 Paulson OB, Strandgaard S, Edvinsson L: Cerebral autoregulation. Cerebrovasc Brain Metab Rev 1990;2:161-192.

22 Ong BY, Greengrass R, Bose D, Gregory G, Palahniuk RJ: Acidemia impairs autoregulation of cerebral blood flow in newborn lambs. Can Anaesth Soc J 1986;33:5-9.

23 Zhang R, Zuckerman JH, Giller CA, Levine $\mathrm{BD}$ : Transfer function analysis of dynamic cerebral autoregulation in humans. Am J Physiol 1998;274:H233-H241.

24 Birch AA, Dirnhuber MJ, Hartley-Davies R, Iannotti F, Neil-Dwyer G: Assessment of autoregulation by means of periodic changes in blood pressure. Stroke 1995;26:834-837.

25 Diehl RR, Linden D, Lucke D, Berlit P: Phase relationship between cerebral blood flow velocity and blood pressure. A clinical test of autoregulation. Stroke 1995;26:1801-1804.
26 Greisen G: Analysis of cerebroarterial Doppler flow velocity waveforms in newborn infants: towards an index of cerebrovascular resistance. J Perinat Med 1986;14:181-187.

27 Liu J, Zhu YS, Hill C, Armstrong K, Tarumi T, Hodics T, Hynan LS, Zhang R: Cerebral autoregulation of blood velocity and volumetric flow during steady-state changes in arterial pressure. Hypertension 2013;62:973-979.

28 Tsuji M, Saul JP, du Plessis A, Eichenwald E, Sobh J, Crocker R, Volpe JJ: Cerebral intravascular oxygenation correlates with mean arterial pressure in critically ill premature infants. Pediatrics 2000;106:625-632.

29 Czosnyka M, Smielewski P, Lavinio A, Pickard JD, Panerai R: An assessment of dynamic autoregulation from spontaneous fluctuations of cerebral blood flow velocity: a comparison of two models, index of autoregulation and mean flow index. Anesth Analg 2008; 106:234-239.

30 Aaslid R, Lindegaard KF, Sorteberg W, Nornes H: Cerebral autoregulation dynamics in humans. Stroke 1989;20:45-52.

31 Massaro AN, Govindan RB, Vezina G, Chang $\mathrm{T}$, Andescavage NN, Wang Y, Al-Shargabi T, Metzler M, Harris K, du Plessis AJ: Impaired cerebral autoregulation and brain injury in newborns with hypoxic-ischemic encephalopathy treated with hypothermia. J Neurophysiol 2015;114:818-824.

32 Howlett JA, Northington FJ, Gilmore MM, Tekes A, Huisman TA, Parkinson C, Chung SE, Jennings JM, Jamrogowicz JJ, Larson AC, Lehmann CU, Jackson E, Brady KM, Koehler RC, Lee JK: Cerebrovascular autoregulation and neurologic injury in neonatal hypoxicischemic encephalopathy. Pediatr Res 2013; 74:525-535.

33 Chalak LF, Tian F, Tarumi T, Zhang R: Cerebral hemodynamics in asphyxiated newborns undergoing hypothermia therapy: pilot findings using a multiple-time-scale analysis. Pediatr Neurol 2016;55:30-36

34 Brady KM, Mytar JO, Kibler KK, Hogue CW Jr, Lee JK, Czosnyka M, Smielewski P, Easley RB: Noninvasive autoregulation monitoring with and without intracranial pressure in the naive piglet brain. Anesth Analg 2010;111: 191-195.

Wavelet Neurovascular Bundle

Dev Neurosci 2017;39:89-96 
35 Lee JK, Yang ZJ, Wang B, Larson AC, Jamrogowicz JL, Kulikowicz E, Kibler KK, Mytar JO, Carter EL, Burman HT, Brady KM, Smielewski P, Czosnyka M, Koehler RC, Shaffner DH: Noninvasive autoregulation monitoring in a swine model of pediatric cardiac arrest. Anesth Analg 2012;114:825-836.

36 Lee JK, Brady KM, Mytar JO, Kibler KK, Carter EL, Hirsch KG, Hogue CW, Easley RB, Jordan LC, Smielewski P, Czosnyka M, Shaffner DH, Koehler RC: Cerebral blood flow and cerebrovascular autoregulation in a swine model of pediatric cardiac arrest and hypothermia. Crit Care Med 2011;39:2337-2345.

37 Roy CS, Sherrington CS: On the regulation of the blood-supply of the brain. J Physiol 1890; 11:85-158.

38 Busija DW, Heistad DD: Factors involved in the physiological regulation of the cerebral circulation. Rev Physiol Biochem Pharmacol 1984;101:161-211.
39 Ariel I, Anteby E, Hamani Y, Redline RW: Placental pathology in fetal thrombophilia. Hum Pathol 2004;35:729-733.

40 Chugani HT, Phelps ME, Mazziotta JC: Positron emission tomography study of human brain functional development. Ann Neurol 1987;22:487-497.

41 Aaslid R: Visually evoked dynamic blood flow response of the human cerebral circulation. Stroke 1987;18:771-775.

42 Roche-Labarbe N, Wallois F, Ponchel E, Kongolo G, Grebe R: Coupled oxygenation oscillation measured by NIRS and intermittent cerebral activation on EEG in premature infants. Neuroimage 2007;36:718-727.

43 Sirota A, Csicsvari J, Buhl D, Buzsaki G: Communication between neocortex and hippocampus during sleep in rodents. Proc Natl Acad Sci USA 2003;100:2065-2069.

44 Torrence C, Webster PJ: Interdecadal changes in the ENSO-Monsoon system. J Climate 1999;12:2679-2690.
45 Lemmers PM, Toet M, van Schelven LJ, van Bel F: Cerebral oxygenation and cerebral oxygen extraction in the preterm infant: the impact of respiratory distress syndrome. Exp Brain Res 2006;173:458-467.

46 Lemmers PM, Zwanenburg RJ, Benders MJ, de Vries LS, Groenendaal F, van Bel F, Toet MC: Cerebral oxygenation and brain activity after perinatal asphyxia: does hypothermia change their prognostic value? Pediatr Res 2013;74:180-185.

47 Tian F, Tarumi T, Liu H, Zhang R, Chalak L: Wavelet coherence analysis of dynamic cerebral autoregulation in neonatal hypoxicischemic encephalopathy. Neuroimage Clin 2016;11:124-132.

48 Brew N, Walker D, Wong FY: Cerebral vascular regulation and brain injury in preterm infants. Am J Physiol Regul Integr Comp Physiol 2014;306:R773-R786. 